\title{
The CMS Event Builder Demonstrator based on Myrinet
}

\author{
G. Antchev, E. Cano, S. Chatelier, S. Cittolin, S. Erhan †, D. Gigi, J. Gutleber, C. Jacobs, \\ F. Meijers, L. Orsini, L. Pollet, A. Racz, D. Samyn, N. Sinanis ††, P. Sphicas
}

CERN, Div. EP, 1211 Geneva 23, Switzerland; †University of California, Los Angeles, USA; $\uparrow \dagger$ ETH Zurich, Switzerland

\section{Abstract}

The data acquisition system for the CMS experiment at the large hadron collider (LHC) will require a large and high performance event building network. Several switch technologies are currently being evaluated in order to compare different architectures for the event builder. One candidate is Myrinet. This paper describes the demonstrator which has been set up to study a small-scalc $(8 \times 8)$ event builder based on a Myrinct switch. Measurements are presented on throughput, overhead and scaling for various traffic conditions. Results are shown on event building with a push architecture.

\section{INTRODUCTION}

The architecture of the data acquisition (DAQ) system for the CMS experiment [1] at the fiture LHC pp collider at CERN is shown Fig. 1. A large high performance network connects 512 readout units (RU's), via a switch fabric, to 512 filter units (FU's). The RU's read out data from detector elements at a first level trigger rate of maximum $100 \mathrm{kHz}$ and buffer the event fragments for up to $1 \mathrm{~s}$. The expected average event size is 1 Mbyte, corresponding to event fragment sizes of 2 kbytes. With events of this size, the $512 \times 512$ event building network requires an effective aggregate bandwidth of $100 \mathrm{Gbytc} / \mathrm{s}$. A software trigger running in the FU's reduces the rate to about $100 \mathrm{~Hz}$ of cvents to record on permanent storage. The event manager broadcasts the first level trigger information to all RU's and performs the event flow control from RU's to FU's.

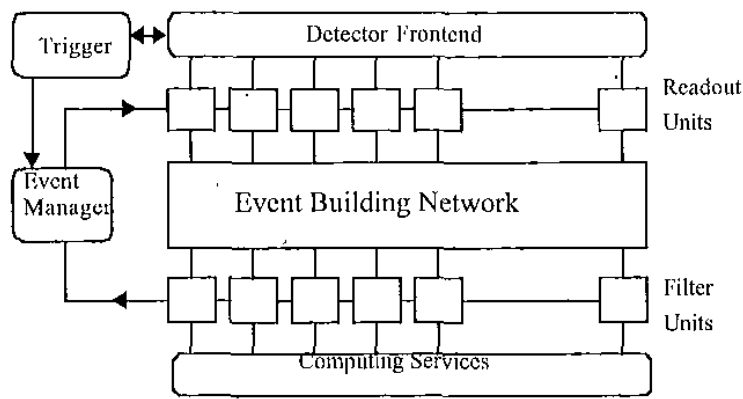

Figure 1: The CMS data acquisition archilecture.

A number of scenarios for cvent flow control have been proposed. In the push architecture, the event manager assigns a destination for each event and broadcasts the event identificr and destination to all sources. The sources then send their event fragments to the assigned destination. In the pull architecture, the destination processor initiates the data transfer by requesting event fragments from each of the sources.
The software trigger can be implemented in multiple levels and may use phased event building, where initially only a subset of the data corresponding to an event are moved and for the fraction of accepted cvents the additional event data are collected. This could reduce the required bandwidth for the event building network by $50 \%$.

Event building traffic is highly systematic as multiple sources compete for the same destination and, depending on the switching technology, the result may be reduced throughput, increased latency and/or loss of data. These effects can be minimised by an appropriate destination assignment algorithm and traffic shaping. Traffic shaping controls the traffic before submission to the network. One technique is the barrel shifter scheme. Hore, sources are synchronised to emit fragments in time slots in such a way that no two sources send to the same destination during the same time slot and all sources regularly send to all destinations in a cycle. This is very efficient for fixed size fragments. Another technique is rate division, where the total link input bandwidth is equally divided between destinations.

Before producing the design of the CMS DAQ system, small-scale prototypes (demonstrators) are being developed to evaluate technologies and study the functionality and performance of the various alternative designs. For event building, earlier work has been reported on Fibre Channel and ATM [2] and on simulations studies [3]. This paper reports on results obtained with a $8 \times 8$ Myrinet event builder. So far, single level event building has been studied with a push architecture. Myrinet is a cost-effective Gbit/s packet-switched network employing crossbars. The goal is twofold: the evaluation of the Myrinet technology and its applicability to event building traffic and also the study of generic event building architectures and protocols.

\section{MYRINET}

Myrinet is a Gbit/s network composed of network interface cards (NICs), connected to crossbar switches by point-to-point links. It is used within concurrent and parallel supercomputers. Myrinet is specified at the data link and physical level as an ANSI standard[4]. Myrinet products are manufactured by Myricom[5]

\section{A. Network Technology}

$\wedge$ Myrinet packet consists of a sequence of bytes starting with a routing header, followed by an arbitrary length payload and terminated by a trailer that includes a CRC byte computed on the entire packet. The routing header is used by the switches, which strip these header bytes to be used to steer the packet through the network. 
A Myrinct link is byte-wide and full duplex with a speed of $160 \mathrm{Mbyte} / \mathrm{s}^{1}$ in each direction. Communication is reliable with very low bit error rates (below $10^{-15}$ ). Since the packet traffic does not share a single physical medium, a Myrinct network is, in principle, scalable.

The non-blocking crossbar switches employ wormhole routing. The routing decision is made as soon as the packet header arrives, the stripped header is then sent to the chosen output link and the rest of the packet follows without being internally buffered. The packet header creates a temporary circuit (wormhole), which closes as the trailer passes through each device. Thus, a worm can stretch across many nodes and links at any time. Wormhole routing minimises latency and buffering requirements compared to switches using store and forward techniques. It also has the advantage that it allows arbitrary length packets.

When the desired output port is not available, the worm is stalled and this information is propagated upstream using backpressure flow control. This flow control is basically STOP/GO and utilises a small (46 byte) 'slack' buffer to absorb the reaction delay.

The performance of the network can be limited by head-ofline blocking. When several packets are contending for the same output link and a packet is stalled because the required output link is busy, all packets in the input queue behind it ane also stalled, cven if their selected output link is free.

\section{B. Network Interface Card}

Figure 2 shows the block diagram of the Myrinet PCI network interface card. The interface is composed of a control processor called LANai with I Mbyte of SRAM. The SR $\Lambda M$ serves as the network buffer memory and also as the codc and data memory of the LANai processor. There are three DMA engines on the NIC: two for data transfers between the network and the SRAM, and one for moving data between the SR $\triangle M$ and the host main memory over the $\mathrm{PCl}$ bus.

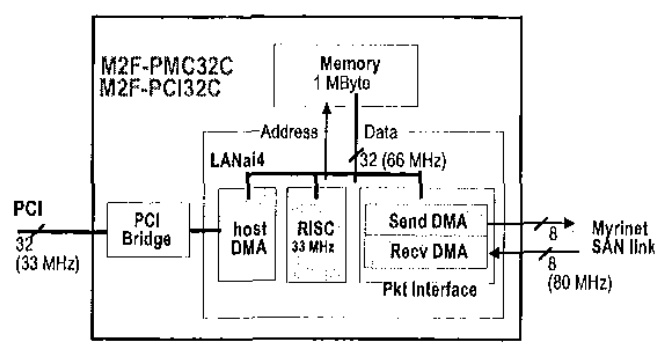

Figure 2: Block diagram of the Myrinet network interface card.

The LANai processor executes a Myrinet control program (MCP) which supervises the operation of the DMA cngines and implements a low-level communication protocol. The internal bus clock runs at twice the PCl clock allowing two DMA engines to operate concurrently at full speed.

\section{THE EVENT BUILDER DEMONSTRATOR}

11 Mbyte is defined as $10^{6}$ bytes.
A demonstrator has been set up to study a small-scale event builder based on Myrinet. All tests were done with a $\mathrm{NxN}$ topology, where the number of sources and destinations are equal $(\mathrm{N})$, and $\mathrm{N}$ is set to 1,4 or 8 .

The configuration of the demonstrator is shown in Fig. 3. A Myrinet switch (M2M-OCT-SW8) connects 8 sources and 8 destinations. The sources are Motorola MVME2306 VMEbus single board computers running VxWorks. The destinations atc SUN Ultra5 PCl-based workstations running Solaris. The sources and destinations act as cmulators of the readout units and filter units, respectively. These nodes arc connected to the Myrinet network with $32 \mathrm{bit} / 33 \mathrm{MHz} \mathrm{PMC}$ and $\mathrm{PCI}$ interfaces (M2F-PMC32C, M2F-PCI32C), respectively. The function of the event manager is performed by an additional SUN workstation. The event flow control messages are transmitted over two separate $100 \mathrm{Mbit} / \mathrm{s}$ Ethernet switched networks, onc connected to the sources and the other to the destinations. The software emulating the $R U$ is derived from the full RU software currently under development [6].

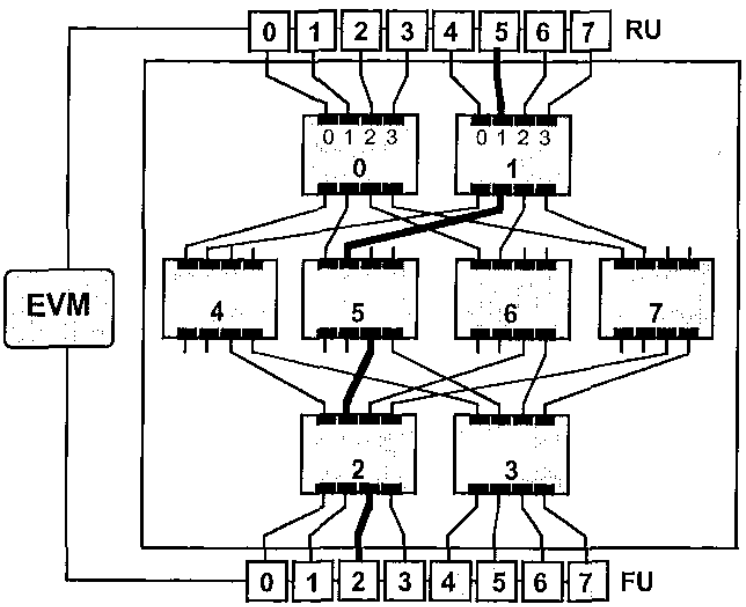

Figure 3: Demonstrator layout with the composite switch, RU sources, FU destinations and an event manager (EVM). The intermal switch structure shows the crossbar topology and numbering scheme. The bold line shows the path from source 5 to destination 2 via the intermediatc crossbar switch 5 according to the algorithm IS $=4+\operatorname{PN}(\operatorname{scc} t e x t)$.

The standard MCP softwate provided by Myricom did not provide the full functionality required for our application. Hence, a custom MCP and associated device drivers for the VxWorks and Solaris hosts have becn developed implementing a low-level communication layer. This message-passing layer implements a circular buffer of message descriptors and associated data, both resident in the NIC memory. It provides reliable and in-order delivery with $\mathrm{CRC}$ error detection ${ }^{2}$. The DM $\Lambda$ from host memory on the PCI bus to the NIC SR $\Lambda M$ has been suppressed, because it would limit the overall performance due to limitations imposed by the available

2 In our tests no single CRC crror was detected after more than 100 hours of running, corresponding to $10^{14}$ bytes transmitted. 
hosts ${ }^{3}$. Instead, the user header needed for the event building protocol is accessed by the host with programmed I/O and we suppress copying the event fragment user data between NIC buffer memory and the host. This enables us to load the Myrinet switching network to the maximum possible extent.

The M2M-OCT-SW8 is a composite switch with 32 external ports comprising eight 8-port crossbars. It is important to study a composite switch, since it is very likely, that the large switch fabric needed for CMS will have to be built by cascading smaller ones. A switch composed of smaller crossbars does not have the same bchaviour as a switch consisting of a single larger crossbar, because of potential internal blocking. Figure 3 shows the internal switch structure and the way it is connected in our configuration. Nlthough Myrinet links are full duplex, in the tests described below, the network is operated in one direction only.

The routing of a packet through the 3-layer switch is fixcd to a unique path for each source-destination pair. The algorithm used relates the intermediate switch number IS to the port number of the source switch PN (see Fig. 3) according to

IS $=4+\mathrm{PN}$

Although alternate paths could have been used at run time, the motivation for a unique path is to avoid unintended randomisation.

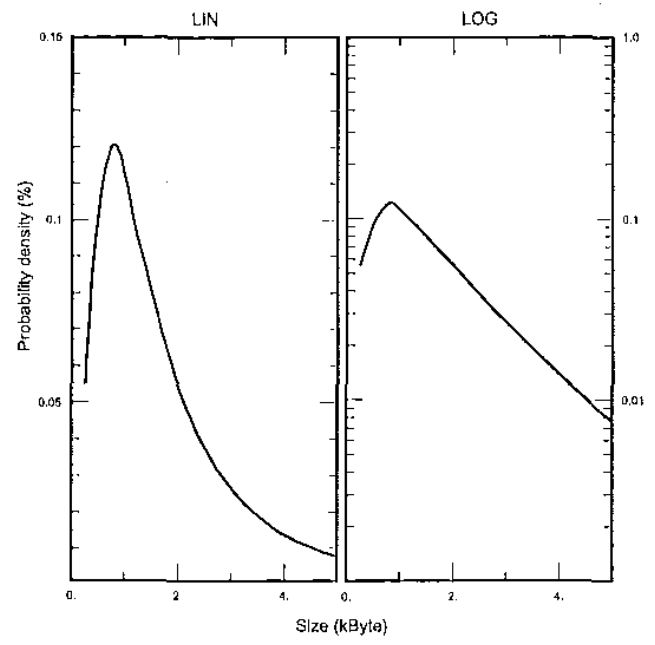

Figure 4: Fragment size distribution for variable size tests (linear and $\log$ scale) for parameter values set to average $=2$ kbyte and RMS $=2$ kbyle :

Tests are made with both fixed and variable size event fragments. Variable size event fragments are generated to mimic the sizes expected for CMS readout. The sizes are gencrated according to the log-normal distribution for various

\footnotetext{
3 It has been verified, that on a PC platform the NIC can perform DMA over PCI bus close to the PCI hardware limit.
}

parameter settings. As an example, Fig. 4 shows the frequency distribution for an average size of 2 kbytes and an $\mathrm{RMS}^{4}$ of 2 kbytes, which is belicved to be approximate to CMS data taking. Note that the distribution is asymmetric, with the most probable value (about 1 kbytc) significantly different from the average value.

\section{RESULTS}

Results are presented on throughput measurements for various test configurations and traffic conditions. These are obtained by runs of typically 10 minutes, corresponding to a very large number of transfers. For each run, the total volume of data transmitted is measured at each source and destination.

\section{A. Point-to-Point Traffic}

The basic performance of the NIC and switch was measured with a number of point-to-point tests. In these tests there is a fixed source-destination assignment, throughout the run.

Test $1: A$ single source sends fixed size packets to a single destination through the switch. The measured throughput and corresponding rate are presented in Fig. 5. An asymptotic throughput of $132 \mathrm{Mbyte} / \mathrm{s}$ is achieved and a $50 \%$ efficiency for a packet size of about 500 bytes.

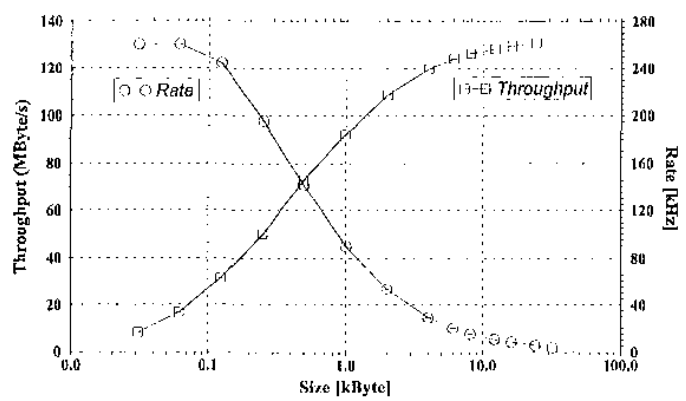

Figure 5: Throughput and corresponding rate versus packet size for $1 \times 1$ configuration.

The transfer time of a packet calculated from the rate, is well described by the linear relationship:

$$
\text { time = overhead + size / speed. }
$$

$\Lambda$ fit yields an overhead of about $4 \mu$ s and a speed of 132 Mbyte/s. The overhead is attributed to processing time of the $\mathrm{MCP}$ in the NIC. The speed of $132 \mathrm{Mbyte} / \mathrm{s}$ is the hardware limit, determined by the NIC memory to link DMA (33 MHz, $32 \mathrm{bi}$ ts). Note that the transfer time is not limited by the link speed $^{5}$ of $160 \mathrm{Mbyte} / \mathrm{s}$. The throughput of this $|x|$ configuration will be referred to as the full performance per source or destination, as it is not affected by traffic conditions.

Test 2; The scaling belraviour is studied for $\mathrm{NxN}$ configurations. Here, there are $\mathrm{N}$ concurrent transfers, with

4 root mean square

5 The packet interface inside the LANai processor inserts 'IDLE' control cycles onto the link if the next data byte is not available. 
mutually exclusive paths, where source $i$ sends to destination $i$ $(i=0 . . \mathrm{N}-1)$. The aggregate throughput for a number of fragment sizes is shown in Fig. 6 for $N=1,4,8$. The throughput scales linearly with the size of the configuration (as expected given the mutually exclusive paths set up for this test).

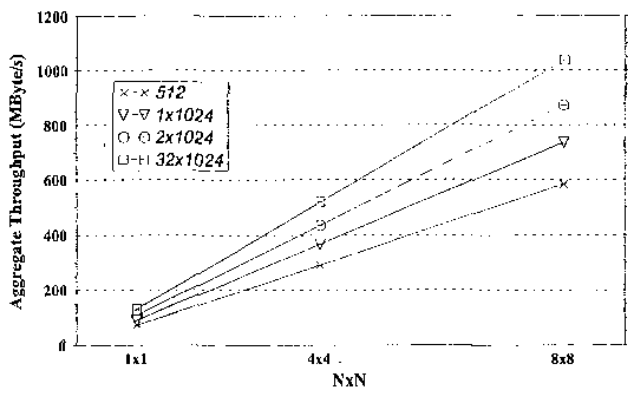

Figure 6: Aggregate throughput for mutually exctusive transfers in $\mathrm{NxN}$ configurations versus $\mathrm{N}$ for fragment sizes of 512,1024 , $2 \times 1024,32 \times 1024$ bytes.

Test 3: The effect of output blocking and of internal blocking is examined. To this end, we measure the throughout at each source for various source $\rightarrow$ destination assignments $s(i j k l m n o p) \rightarrow d\left(i^{\prime} j^{\prime} k^{\prime} l\right.$ l'm $\left.^{\prime} n^{\prime} o^{\prime} p '\right)$, where source $z$ sends to destination $z^{\prime}(\mathrm{z}=i . p)$. The case $s(01234567) \rightarrow d(01234567)$ corresponds to test 2 , discussed above. The throughput measured at source $\# 0$ is shown in Fig. 7 for various destination assignments.

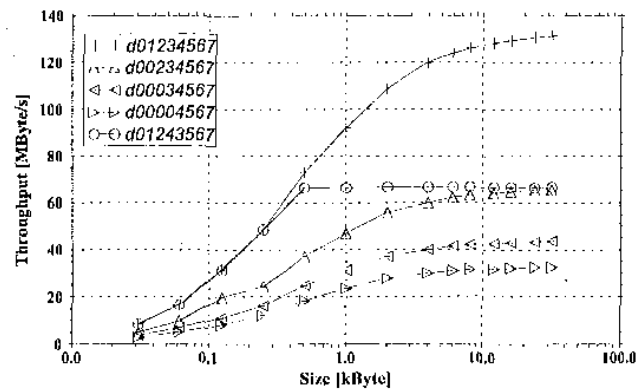

Figure 7: Throughput versus packet size at source $\# 0$ for various destination assignments in an $8 \times 8$ configuration.

Output blocking occurs if more than one source sends simultancously to the same destination. $\Lambda \mathrm{n}$ example of such a clash is the destination assignment $s(01234567) \rightarrow$ $d(00234567), d(00034567)$ and $d(00004567)$. The observed throughput at source $\# 0$ is reduced with respect to $s(01234567)$ $\rightarrow d(01234567)$ by a factor 2,3 and 4 , respectively. Thus, the bandwidth sharing at the output is fair. It has been verified that the throughput of the other sources, i.e. the ones not sending to destination $\# 0$, remains unaffected.

For the $8 \times 8$ configuration, internal blocking occurs if sources connected to crossbar \#0 share an intermediate path to the destination crossbar with sources connected to crossbar $\# 1$ (see Fig. 3). This can be provoked with the destination assignment $s(01234567) \rightarrow \mathrm{d}(01243567)$, where sources 3 and 4 are permuted and $0 \rightarrow 0$ and $4 \rightarrow 3$ contend for the crossbar 4 to 2 connection. The throughput at source $\# 0$ is reduced by a factor two and is equally shared 6with source \#4. The same holds for sources $\# 3$ and $\# 7$. It has been verified that the throughput of the remaining sources remains unaffected.

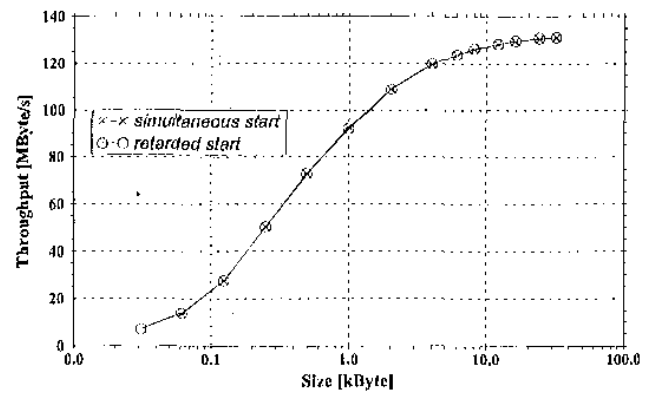

Figure 8: Throughput versus fragment size at FU destination \#0 for event building traffic in the $4 \times 4$ configuration without event manager.

\section{B. Random Traffic}

The behaviour of the switch under random traffic has been measured with the same $8 \times 8$ configuration. Here, each source scnds, independently, fixed-size packets to a randomly chosen destination. The measured throughput was about $60 \%$ of the full performance. This is because the throughput of the network under random traffic is limited by head-of-line blocking. An analytical model for this configuration gives $58 \%$ of the cross section bandwidth, which is in reasonable agreement with our measurement.

\section{Event Building Traffic}

These tests are done without the event manager. Therefore, there is no external event flow control. It is interesting to investigate, whether the back-pressure flow control in Myrinet will bring the switch traffic in a stablc equilibrium close to full performance.

The RU sources generate event fragments of fixed size with event identifier evtid and send these to the FU destinations according to the round robin assignment

FU\# = evtid $\bmod \mathrm{N}$.

Each event fragment is sent as a single packet.

$\Lambda \mathrm{fter}$ initial broadcast of the start command, the sources are essentially running freely, only moderated by the back pressure mechanism. Two initial start conditions are tested; in the simultaneous start, all RU's start sending fragments immediately after receiving the start command. In the retarded start, RU \#n skips the first $n$ events. This will start the traffic in the barrel shifter mode.

6 The observation that the throughput is constant down to about 500 bytes can be explained by the fact that both source and destination operate at $50 \%$ of the nominal rate and overhead from one source can be overlapped with transfer of the other. 
Results arc shown in Fig. 8 and 9 for these two initial start conditions for the $4 \times 4$ and $8 \times 8$ configurations, respectively. The throughput versus fragment size is shown for $\mathrm{FU} \# 0$. It has been verified that it is the same for all FU's .

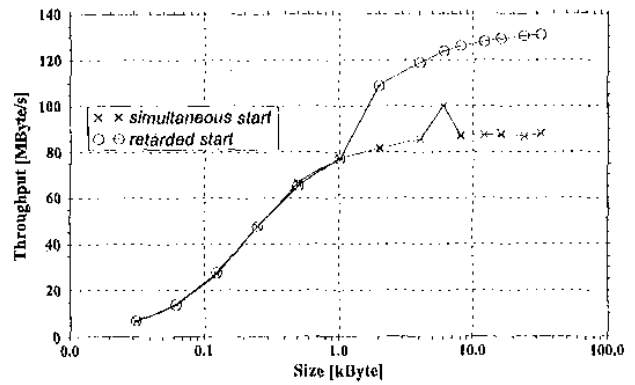

Figure 9: Throughput versus fragment size at $\mathrm{FU}$ destination \#0 for event building traffic in the $8 \times 8$ configuration without event manager.

The $4 \times 4$ configuration is equivalent to an actual $4 \times 4$ crossbar. Both initial start conditions give throughput close to full performance (Fig, 8). This is expected because after a few initial cycles the traffic evolves to a barrel shifter, irrespective of the initial condition.

For the $8 \times 8$ configuration (Fig. 9), it can be scen that the retarded start locks the traffic into a mode close to full performance, whereas for the simultaneous start, it locks itsclf into a non-optimal mode. The latter is due to the internal blocking and depends on the destination assignment algorithm. A quantitative understanding of the non-optimal modes requires some further tests with different parameters and it will be interesting to compare these measurements with a detailed simulation.

\section{Event Building with Event Manager}

Event flow control is implemented with the event manager in the following way. The event manager receives and collects cvent requests from the destinations, assigns the next event to a destination in a round robin fashion and broadcasts this information to all sources, which subsequently send the corresponding event fragments through the Myrinet network. For efficiency rcasons, several requests are grouped in an Ethernet packet. The number of requests is always a multiple of the number of destinations $\mathrm{N}$, up to a maximum of 352 , imposed by the Ethernet packet length. The RU sources apply a kind of barrel shifter traffic shaping whereby RU \#n starts with the $n$ 'th request in the packet and cycles through the list.

Measurements were done for the $8 \times 8$ configuration with fixed-size fragments. The throughput and event rate versus fragment size are shown for FU \#0 in Fig. 10. Note that the rate of assembled events per destination scales as $1 / \mathrm{N}$, because each destination receives 1 in $N$ events, while the rate of event fragments is invariant. The achieved throughput is close to full performance, with a small degradation for small fragment sizes induced by additional event manager overhead. The rate of assembled events at a single destination (FU \#0) is about 6.5 $\mathrm{kHz}$ for the nominal 2 kbyte fragment size. As there are 8 destinations, this corresponds to a level 1 trigger rate of about $50 \mathrm{kHz}$.

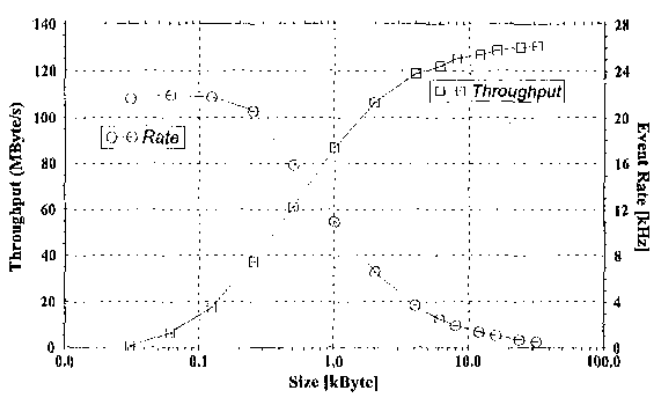

Figure 10: Throughput and rate versus fragment size at FU \#0 for the $8 \times 8$ configuration with event manager. The rate is the number of events per second assembled at the single destination.

The effect of variable size event fragments has also been studied. Here, each source sends, independently, fragments with sizes generated according to a distribution mimicking that expected in the CMS readout (sce section III). Measurements have been done for different combinations of average size and RMS of this distribution. Figure 12 shows the throughput versus average size for a number of different RMS values, compared to the fixed size case.

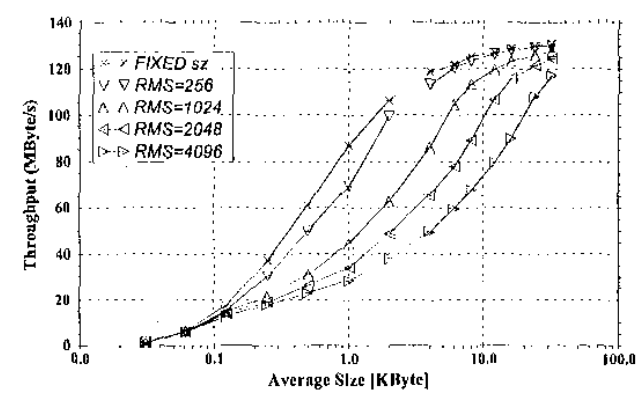

Figure 11: Throughput versus average size for RMS values of 256, 1024,2048 and 4096 bytes. The fixed size case is also plotted. Measurements are done at FU $\# 0$ for the $8 \times 8$ configuration with event manager.

A considerable degradation of throughput with respect to fixed size is obscrved. This is due to head-of-line blocking. For the nominal average fragment size of 2 kbyte, the throughput reduces from $109 \mathrm{Mbytes} / \mathrm{s}$ for fixed size to $54 \mathrm{Mbyte} / \mathrm{s}$ for a RMS sprcad of 2 kbyte.

\section{FUTURE WORK}

The performance degradation for event fragments of variable sizc can be overcome by grouping several event fragments into large fixed-size packets and operating the system as a barrel shifter. $\Lambda \mathrm{n}$ alternative could be a rate division algorithm, similar to the ATM constant bit rate mechanism. Here, event fragments are split into small size packets and the source 
cycles over all destinations. We will study the feasibility of [4] ANSI/VITA 26-1998, Myrinet-on-VME protocol specification. such a scheme in the case of Myrinet.

In the near future, the demonstrator will be expanded from a $8 \times 8$ to a $16 \times 16$ configuration, and the source and destination nodes will be gradually replaced by personal computers. Furthermore, we will introduce the new 64-bit based Myrinet NIC's, which should allow to operate the network at the full link specd of $160 \mathrm{Mbytc} / \mathrm{s}$. The same test bed will be used later to cvaluate GigaBit Ethernet.

So far, event building was studied with a push architecture. This will be extended to the pull scenario and also to phased event building.

\section{CONCLUSIONS}

The CMS event builder demonstrator based on Myrinet has been presented. The aim of the study is to cvaluate the Myrinet technology itself and to examine event building architectures in general. The use of Myrinet as network technology enabled us to assemble a high performance test system within a few months. It also has enabled us to assess the impact of overheads on overall performance.

Throughput measurements were done on the $8 \times 8$ event builder for point-to-point, random and event building traffic. The composite switch behaves as expected. High efficiency can be achieved for large packets and appropriate traffic shaping, such as the barrel shifter.

Results have been presented on event building with a push architeclure. For fragment sizes of 2 kbytes, the achieved trigger rate is about $50 \mathrm{kHz}$. As expected, substantial performance degradation occurs for event fragments of variable size. A possible mechanism to overcome this is the grouping of scveral event fragments into fixed-size packets. In principle, the present Myrinct hardware could satisfy the CMS requirements within a factor two, assuming scaling to a large (500 by 500 ) system.

The development of the behavioural simulation of the Myrinet NIC and switches in the existing framework is in progress. The measurements obtained from the demonstrator provide the necessary parameters needed in order to make predictions for large-scale systems.

These studies are part of an ongoing program of work on the evaluation of event building architectures and switch technologics. They are expected to conclude in a first comprehensive design for the CMS DAQ in 2001, in time for the Technical Design Report.

\section{REFERENCES}

[1] The CMS Collaboration, The Compact Muon Solenoid, CERN, Technical Proposal, No. 7, LIICC 94-38 December 1995.

[2] T.Ladzinski, A. Jusko, N. Lejeune, D. Samyn, CMS Data Links and Event Builder Studies, $3^{\text {rd }}$ Workshop on Electronics for LIC Experiments London, UK; 22-26 Sep 1997. Publ. In: Proceedings CERN, Geneva, 1997 CERIB-LHCC-97-60 (450454).

[3] N.J. Sinanis, et al., Performance Evaluation of the CMS DAQ System using Simulations, Computing in ligh Energy and Nuclear Physics Conference (CHEP98), Chicago Illinois, 1998.
[5] Myricom, Inc., Arcadia, CA, USA. http:://www.myri.com

[6] G. Antchev et al., A Software Approach for Readout and Data Acquisition in $C M S$, This conference. 